\title{
Materials Selection Using a 2-tuple Linguistic Multi-criteria Method
}

\author{
Dalmarino Setti $^{a}{ }^{\circledR}$, Maria Nalu Verona ${ }^{a}$, Bruno Bellini Medeiros ${ }^{a}$, Alex Restelli ${ }^{b}$ \\ ${ }^{a}$ Departamento de Mecânica - DAMEC-PB, Universidade Tecnológica Federal do Paraná - UTFPR, \\ Via do Conhecimento, km1, 85503-390, Pato Branco, PR, Brasil \\ ${ }^{b}$ Instituto Federal de Educação, Ciência e Tecnologia de Santa Catarina, Campus São Lourenço do Oeste, \\ Rodovia SC-480, s/n, Distrito Frederico Wastner, 89994-000, São Lourenço do Oeste, SC, Brasil
}

Received: December 12, 2018; Revised: August 13, 2019; Accepted: Octuber 10, 2019

\begin{abstract}
The materials selection can affect the design component radically, with effect on the manufacturing systems efficiency, environmental impact issues, and customer satisfaction. There are different methods employed for materials selection; however, two steps are usual for most of these methods: screening and ranking. The ranking step identifies among materials candidates those that can perform the function the best as possible. Multi-criteria methods have been widely employed to materials selection, especially in the ranking step. Most of these methods take advantage of fuzzy numbers and linguistic variables to process qualitative information and information with uncertainties. One of the approaches that have been developed to solve issues related to make decisions in multi-criteria methods using linguistic information is the 2-tuple linguistic computational model. The main advantage of this approach is taking the "loss of information" away, which provides a higher precision on results. This paper aims to present a multi-criteria method for materials selection ranking step based on 2-tuple linguistic variables. The steps and several equations needed to apply the proposed method are described. Two case studies are presented and compare results with other methods to demonstrate the proposed method potential.
\end{abstract}

Keywords: Materials Selection; Multi-criteria Method; Linguistic 2-tuple Variables

\section{Introduction}

The materials selection is an essential part of new products development process ${ }^{1}$ and shows interdependence either shape design or manufacturing process ${ }^{2,3}$. Therefore, the selection of the material can radically affect the component's design, also affecting the manufacturing systems efficiency, the environmental impacts, and customer satisfaction.

There are different methods employed for materials selection; however, two steps are usual for most of these methods: screening and ranking ${ }^{4}$. Multi-criteria methods have been widely applied to materials selection, especially in the ranking step ${ }^{5-7}$. Several of these methods take advantage of quantitative data ${ }^{8-10}$, in a while, others make use of qualitative data presented as fuzzy numbers ${ }^{11,12}$ or linguistic variables ${ }^{13}$. Moreover, some methods allow qualitative and quantitative data processing simultaneously ${ }^{14,15}$.

Using linguistic variables on materials selection enables obtaining materials performance experts opinions from materials performance experts and provide a direct way of managing uncertainties and modeling qualitative assessments $^{16}$. One of the approaches that have been developed to solve issues related to make decisions in multicriteria methods using linguistic information is the 2-tuple linguistic computational method ${ }^{17}$, the main advantage of this approach is taking the "loss of information" away, which provides a higher precision on results ${ }^{16}$.
This paper aims to present a multi-criteria method to materials selection ranking step based on 2-tuple linguistic variables. The main contribution from the approach proposed is the possibility of processing quantitative information as linguistic variables, with different linguistic variable scales, which allows realizing the desired goals to materials selection and to perform the preferences of decisions makers by criteria weights and carry out the processing by 2-tuple linguistic variables.

The paper was divided into three main sections. In section 2 are described the main fundaments of the 2-tuple linguistic method, which are needed to the proposed method application and its steps are presented in section 3 . In section 4, two materials selection case studies are performed to validate and exemplify the proposed method.

\section{The 2-tuple Linguistic Model}

A 2-tuple linguistic variable is represented as $\left(s_{i}, \alpha\right)$, where $s_{i}$ is a linguistic variable and $\alpha$ is a numeric value been a symbolic conversion of this term ${ }^{12,13}$. Be $S=\left\{\mathrm{s}_{0}, \ldots, \mathrm{s}_{\mathrm{g}}\right\}$ the linguistic terms set and e $\tilde{S}=\mathrm{S} \times[-0,5,0,5)$ the 2-tuple terms set associated. The function $\Delta$ is defined by Herrera and Martínez ${ }^{17}$ :

$$
\begin{aligned}
& \Delta:[0, g] \rightarrow \tilde{S}, \\
& \Delta(\beta)=\left\{\begin{array}{l}
S i, \\
\alpha=\beta-i, \alpha \in[-0.5,0.5),
\end{array}\right.
\end{aligned}
$$


For exemplify, the function $\Delta$ follow the example suggested by $\mathrm{Xu}^{18}$.

Example 1: For $S_{1}=\left\{s_{0}, s_{1}, s_{2}, s_{3}, s_{4}, s_{5}, s_{6}\right\}$ and $\beta=5.5$ be the value of simbolic aggregation. Then: $\Delta(\beta)=\Delta(5.5)$ $=\left(s_{6},-0.5\right)$

For the linguistic assessment scale $S_{1}$ and the 2-tuple ( $s_{i}$, $\alpha$ ) there is always a function $\Delta^{-1}$, such that, from the 2-tuple $\left(s_{i}, \alpha\right)$, it returns its equivalent numeric value $\beta \in[0, g]$ :

$$
\begin{aligned}
& \Delta^{-1}: \tilde{S} \rightarrow[0, g], \\
& \Delta^{-1}\left(S_{i}, \alpha\right)=i+\alpha .
\end{aligned}
$$

For exemplify, the function $\Delta^{-1}$ follows other example suggested by $\mathrm{Xu}^{18}$.

Example 2: For $S_{1}=\left\{s_{0}, s_{1}, s_{2}, s_{3}, s_{4}, s_{5}, s_{6}\right\}$ and the 2-tuple $\left(s_{i}, \alpha\right)=\left(s_{4}, 0.3\right)$. Then: $\left(s_{i}, \alpha\right)=\Delta^{-1}\left(s_{4}, 0.3\right)=4+0.3=4.3$

The linguistic terms computational processing is carried out using the operator based on $\Delta$ and $\Delta^{-1}$ functions, these operations include unification of information, and ranking, which are the steps of the proposed model at section 3 .

\section{Materials Selection Method with 2-tuple Linguistic Variables}

The proposed method in this paper is a compensatory multi-criteria method. In this type of method, the changes in one criterion can be compensated for different variations in any other criteria ${ }^{19}$. The proposed method selects the alternative that represents the highest score, comparing the materials candidates taking in an account the established targets for each criterion (Step 4) and criteria relevance (weights) appointed by the decisions makers involved in the materials selection process.

In the evaluation, the alternatives are arranged in $i$ rows, and the evaluated criteria are allocated in $j$ columns.

In the developed proposed method are needed the following steps:

Step 1: Unification and Conversion. This step has the aim of gathering all data in the same linguistic set; for this reason, the appropriate form of unification and conversion is presented.

1.1 Unification: Herrera and Martíne $z^{17}$ suggested in the case with will the focus in the use of linguistic information for modeling performance evaluations, we have to choose the appropriate linguistic descriptors for the term set and their semantics. To accomplish this objective, an important aspect to analyze is the granularity $(g)$ of information, i.e., the cardinality of the term set.

It is usual to find out different linguistic assessment sets in the literature, by issues of preference or needs; thus, the result from information that the decisions makers provide can be from different granularity, i.e., linguistic sets with a different number of linguistic variables. The adopted method for these cases is called scale unification and follows the methodology used by Herrera, Herrera-Viedma, and Martínez; and Martínez and Herrera; ${ }^{21,22}$, where the transformation functions are applied to conduct linguistics information to numeric and again as usual linguistic format, unified. For more details, consult Martinez, Liu, Yang J-B, et al..$^{23}$ between this transformation function was recursively generalized to transform linguistic terms between any linguistic level in the linguistic hierarchy.

As Martinez, Liu, Yang J-B, et al. ${ }^{23}$ we assume that levels containing linguistic terms are triangular shaped, symmetrical, and uniformly distributed. In addition, the linguistic term sets have an odd number of linguistic terms being the middle one the value of indifference.

To choose a basic set of linguistic terms, $R_{\mathrm{u}}=\left\{r_{0}, r_{1}, \ldots, r_{t}\right\}$ to, $\mathrm{S}_{\mathrm{t}}=\left\{s_{0}, s_{1}, \ldots, s_{g}\right\}$, one must find the maximum number the terms starting set to keep the uncertainty degree associated with each expert, as well as the discrimination capacity to express the preferred values. The remaining process resolution to unification is carried out applying the following equation:

$$
T F_{t}^{g}\left(s_{i}^{g} \alpha^{g}\right)=\Delta\left(\frac{\Delta^{-1}\left(R_{i}^{t}, \alpha^{t}\right) \cdot g}{t}\right)
$$

$g=$ highest index term of the linguistic set (linguistic hierarchy) of unification.

$t=$ highest index term of the current linguistic set (linguistic hierarchy)

Note: Transformation must occur always from data set with lower quantity to that with a higher quantity of terms, so there is not loss of information.

Example 3: For $R_{1}=\left\{r_{0}, r_{1}, r_{2}, r_{3}, r_{4}, r_{5}, r_{6}\right\}$ and the 2-tuple $\left(s_{i}, \alpha\right)=\left(\mathrm{r}_{4}, 0.4\right)$. Unification this evaluation to $S_{1}=$ $\left\{s_{0}, s_{1}, s_{2}, s_{3}, s_{4}, s_{5}, s_{6}, s_{7}, s_{8}\right\}$ is possible applying equation (3).

$$
\begin{gathered}
T F_{\mathrm{t}}^{\mathrm{q}}\left(s_{\mathrm{i}}^{\mathrm{q}}, \alpha^{\mathrm{q}}\right)=\Delta\left(\frac{\Delta^{-1}\left(S_{\mathrm{i}}^{\mathrm{t}}, \alpha^{\mathrm{t}}\right) \cdot(q-1)}{(t-1)}\right) \rightarrow T F_{7}^{9}\left(S_{\mathrm{i}}^{9}, \alpha^{9}\right)= \\
\Delta\left(\frac{\Delta^{-1}\left(r_{4}, 0.4\right) \times 8}{6}\right) \rightarrow T F_{7}^{9}\left(S_{\mathrm{i}}^{9}, \alpha^{9}\right)= \\
\Delta\left(\frac{4.4 \times 8}{6}\right)=\Delta(5.87)=\left(s_{6},-0.13\right) .
\end{gathered}
$$

1.2 Conversion: The conversion is necessary for situations which one combine linguistic information with some quantitative criteria. In these situations one uses the equation 4 to convert the quantitative values into linguistic variables, the equation 4 is applied when it comes to benefit criteria, i.e., as higher as the material property value concerning this criteria, better. The conversion of quantitative values for costs criteria, which are those that the smaller is the property value, the better it is carried out based on equation 5 .

$$
\begin{aligned}
& \left(s_{i j}, \alpha_{j}\right)=\Delta\left(\left(\frac{x_{i j}}{\operatorname{Maxx}_{i j}}\right) \cdot g\right) \\
& \left(s_{i j}, \alpha_{j}\right)=\Delta\left(\left(\frac{\text { Minx }_{i j}}{x_{i j}}\right) \cdot g\right)
\end{aligned}
$$


Step 3: Decision maker's preferences (Weights of Criteria). The decision-makers determine the relevance of each criterion, i.e., the criteria weights by linguistic variable sets. A unique linguistic vector is obtained by assessments aggregation by equation 3 and a criteria weight vector, as a real number, is obtained by equation 6 and 7 :

$$
\begin{aligned}
\left(w_{j}, \alpha_{j}\right) & =\Delta\left(\frac{1}{k} \sum_{l=1}^{k} \Delta^{-1}\left(w_{k j}, \alpha_{k j}\right)\right) \\
w_{j} & =\frac{\Delta^{-1}\left(w_{j}, \alpha_{w j}\right)}{\sum_{j=1}^{n} \Delta^{-1}\left(w_{j}, \alpha_{w j}\right)}
\end{aligned}
$$

Step 4: Materials Information (Materials performance related to criteria). The decision-makers evaluate the candidate materials performance relating to each one of the criteria by a linguistic variables set. Either qualitative or quantitative information can be applied but must be processed by the first step equations - the single matrix of materials in the rows and criteria in the columns é obtained by equation 8 . Each matrix element represents the material performance (row) relating to criteria (column).

$$
\left(S_{i j}, \alpha_{i j}\right)=\Delta\left(\frac{1}{k} \sum_{l=1}^{k} \Delta^{-1}\left(S_{i j}, \alpha_{i j}\right)\right)
$$

Step 5: Target (Goals). Determining the proposed solution (targets), to each criterion, express by a linguistic variable, the target must be the better performance alternative for each criterion or the material desired value.

Step 6: Ranking. Each candidate material performance is obtained by equation 9,10 , and 11 . It is a target-based normalization adaptation of Jahan et al. ${ }^{24}$. The equation in this step provides the linguistic ranking, and the more suitable material to the intended application is selected.

For that, one can find the maximum value from the alternatives for each criterion, obtained by equation 9 :

$$
\mathrm{L}_{\mathrm{j}}=\operatorname{Max}\left\{\Delta^{-1}\left(\mathrm{~S}_{\mathrm{j}}, \alpha_{\mathrm{j}}\right)^{\max }, \Delta^{-1}\left(\mathrm{~T}_{\mathrm{j}}, \alpha_{\mathrm{j}}\right)\right\}
$$

Moreover, one can find the minimum value from the alternatives for each criterion, obtained by equation 10 :

$$
\mathrm{P}_{\mathrm{j}}=\operatorname{Min}\left\{\Delta^{-1}\left(\mathrm{~S}_{\mathrm{j}}, \alpha_{\mathrm{j}}\right)^{\min }, \Delta^{-1}\left(\mathrm{~T}_{\mathrm{j}}, \alpha_{\mathrm{j}}\right)\right\}
$$

The distance from each alternative to the target can be obtained from these values, and to affect the weight relative to the criterion, considering the sum of these values of each alternative respective to each criterion (from the rows) will provide the indicator that will allow ranking them. For this purpose the equation 11 is used:

$\left(\mathrm{m}_{\mathrm{i}}, \alpha_{\mathrm{i}}\right)=\Delta\left(\mathrm{g}\left(\sum_{\mathrm{j}}^{\mathrm{n}}\left(w_{\mathrm{j}}\right) \cdot\left[1-\frac{\left|\Delta^{-1}\left(\mathrm{~S}_{\mathrm{i}}, \alpha_{\mathrm{ij}}\right)-\Delta^{-1}\left(\mathrm{~T}_{\mathrm{j}}, \alpha_{\mathrm{j}}\right)\right|}{\mathrm{L}_{\mathrm{j}}-\mathrm{P}_{\mathrm{j}}}\right]\right)\right)$

\section{Proposed Method Applications}

Two case studies are presented to demonstrate the materials selection proposed method. The case study 1 adapted from Jeya Girubha and Vinodh ${ }^{12}$ that involves the materials selection of an automotive component (panel) was used to demonstrate and validate the proposed method application. In this case study, four types of polymers SMA, PC, PP, and ABS are alternatives to materials selection. The assessment criteria are as follow: Maximum temperature (C1); Recyclability (C2); Elongation (C3); Weight reduction (C4); Thermal conductivity (C5); Tensile Strength (C6); Cost (C7); Toxicity level (C8).

The linguistic variables set used during the assessment, i.e., material performance was: $\mathrm{S}=\left\{\mathrm{S}_{0}=\right.$ Very Low; $\mathrm{S}_{1}=$ Low; $\mathrm{S}_{2}=$ Low Medium; $\mathrm{S}_{3}=$ Medium; $\mathrm{S}_{4}=$ High Medium; $\mathrm{S}_{5}=$ High; $\mathrm{S}_{6}=$ Very High $\}$.

Decision-making was based on three main sustainable pillars, thereby the material been economical, ecological, and beneficial to society.

On case study 1 development, the method's step 1 was not necessary, because all information was presented at the same linguistic scale and without quantitative values.

Table 1 shows the linguistic weight values calculated by equation 6 and 7 that comprehends the step 2 of the proposed method.

Example 4: Considering 5 decision-makers evaluations regarding the $\mathrm{C} 3$ criterion relevance being: Good; Good; Medium Good; Medium Good; Good; being their respective 2-tuple:applying the equation (5):

$$
\begin{gathered}
\left(w_{j}, \alpha_{j}\right)=\Delta\left(\frac{1}{k} \sum_{l=1}^{k} \Delta^{-1}\left(w_{k j}, \alpha_{k j}\right)\right) \Rightarrow \\
\Rightarrow\left(w_{c 3}, \alpha_{c 3}\right)=\Delta\left(\frac{1}{5} \times(5+5+4+4+5) \Rightarrow\right. \\
\Rightarrow\left(w_{c 3}, \alpha_{c 3}\right)=\Delta(4.6) \Rightarrow\left(w_{c 3}, \alpha_{c 3}\right)=\left(s_{5},-0.4\right)
\end{gathered}
$$

Table 1. 2-tuple linguistic criteria weights and real numbers criteria weighs- Case study 1 - Polymers selection

\begin{tabular}{cccccccccc}
\hline \multirow{2}{*}{ Weights } & \multicolumn{8}{c}{ Criteria } \\
\cline { 2 - 9 } & $\mathrm{C} 1$ & $\mathrm{C} 2$ & $\mathrm{C} 3$ & $\mathrm{C} 4$ & $\mathrm{C} 5$ & $\mathrm{C} 6$ & $\mathrm{C} 7$ & $\mathrm{C} 8$ \\
\hline Equation $(6)$ & $\left(\mathrm{S}_{5}, 0,00\right)$ & $\left(\mathrm{S}_{5}, 0,00\right)$ & $\left(\mathrm{S}_{5},-0,40\right)$ & $\left(\mathrm{S}_{5}, 0,00\right)$ & $\left(\mathrm{S}_{5}, 0,40\right)$ & $\left(\mathrm{S}_{4}, 0,40\right)$ & $\left(\mathrm{S}_{5}, 0,20\right)$ & $\left(\mathrm{S}_{4}, 0,40\right)$ \\
Equation $(7)$ & 0,128 & 0,128 & 0,118 & 0,128 & 0,138 & 0,113 & 0,133 & 0,113 \\
\hline
\end{tabular}


Example 5: Taking the unified data from Table 1 to obtain the normalized weight relative to $\mathrm{C} 5$, using the equation (6): $w_{\mathrm{j}}=\frac{\Delta^{-1}\left(w_{\mathrm{j}}, \alpha_{\mathrm{wj}}\right)}{\sum_{\mathrm{j}=1}^{\mathrm{n}} \Delta^{-1}\left(w_{\mathrm{j}}, \alpha_{\mathrm{wj}}\right)} \Longrightarrow w_{\mathrm{c} 5}$

$=\frac{\Delta^{-1}\left(s_{5}, 0.4\right)}{\Delta^{-1}\left(\left(s_{5}, 0\right)+\left(s_{5}, 0\right)+\left(s_{5},-0.4\right)+\left(s_{5}, 0\right)+\left(s_{5}, 0.4\right)+\left(s_{4}, 0.4\right)+\left(s_{5}, 0.2\right)+\left(s_{4}, 0.4\right)\right)} \Longrightarrow$
$\Longrightarrow w_{c 5}=\frac{\Delta(5.4)}{\Delta(5+5+4.6+5+5.4+4.4+5.2+4.4)} \Longrightarrow w_{\mathrm{c} 5}=\frac{\Delta(5.4)}{\Delta(39)} \Longrightarrow w_{\mathrm{c} 5}=0.138$

The assessments provided by experts after aggregation by equation 8 , Step 3 , are presented at Table 2 .

Example 6: Considering 5 decision-makers evaluations concerning to Alternative ABS performance on $\mathrm{C} 2$ criterion as: Very High, High, Very High, High, Very High; being it's respective 2-tuple: $\left(s_{6}, 0\right) ;\left(s_{5}, 0\right) ;\left(s_{6}, 0\right)\left(s_{5}, 0\right) ;\left(s_{6}, 0\right)$, the equation (8) is used:

$$
\begin{gathered}
\left(S_{i j}, \alpha_{i j}\right)=\Delta\left(\frac{1}{k} \sum_{l=1}^{k} \Delta^{-1}\left(S_{i j}, \alpha_{i j}\right)\right) \Rightarrow \\
\Rightarrow\left(S_{A B S, C 2}, \alpha_{A B S, C 2}\right)=\Delta\left(\frac{1}{5} \times(6+5+6+5+6)\right) \Rightarrow \\
\Rightarrow\left(S_{A B S, C 2}, \alpha_{A B S, C 2}\right)=\Delta(5.6) \Rightarrow\left(S_{A B S, C 2}, \alpha_{A B S, C 2}\right)=\left(s_{5}, 0.6\right)
\end{gathered}
$$

In the step 4 is determined the intended targets for each criteria, in case study 1 was used the alternative with better performance compared to each criteria as target to achieve. The target set to eight criteria is the follow: $\mathrm{M}=\left\{\left(\mathrm{s}_{5}, 0,25\right) ;\left(\mathrm{s}_{6}, 0,00\right)\right.$; $\left.\left(\mathrm{s}_{6}, 0,00\right) ;\left(\mathrm{s}_{6}, 0,00\right) ;\left(\mathrm{s}_{5}, 0,50\right) ;\left(\mathrm{s}_{5}, 0,50\right) ;\left(\mathrm{s}_{6}, 0,00\right) ;\left(\mathrm{s}_{6}, 0,00\right)\right\}$.

Step 5 is performing by equations 9,10 , and 11 is presented in Table 3.

Example 7: Taking the performance of the alternatives from column $\mathrm{C} 3$ on table 2, considering the decision-makers desired goal related to $\mathrm{C} 3$ criterion performance, being its respective 2-tuple as:, the alternative $\mathrm{PC}$ score relative to C3 criterion can be obtained from the equations (9, 10 e 11):

$$
\begin{gathered}
L_{\mathrm{j}}=\operatorname{Max}\left\{\Delta^{-1}\left(S_{\mathrm{j}}, \alpha_{\mathrm{j}}\right)^{\max }, \Delta^{-1}\left(T_{\mathrm{j}}, \alpha_{\mathrm{j}}\right)\right\} \Rightarrow \\
\Rightarrow L_{\mathrm{c} 3}=\operatorname{Max}\left\{\Delta^{-1}\left(s_{5}, 0.4\right), \Delta^{-1}\left(s_{5}, 0.4\right)\right\} \Longrightarrow L_{\mathrm{c} 3}=\left(s_{5}, 0.4\right) \\
P_{\mathrm{j}}=\operatorname{Min}\left\{\Delta^{-1}\left(S_{\mathrm{j}}, \alpha_{\mathrm{j}}\right)^{\min }, \Delta^{-1}\left(T_{\mathrm{j}}, \alpha_{\mathrm{j}}\right)\right\} \Rightarrow \\
\Rightarrow P_{\mathrm{c} 3}=\operatorname{Min}\left\{\Delta^{-1}\left(s_{4}, 0.2\right), \Delta^{-1}\left(s_{5}, 0.4\right)\right\} \Longrightarrow P_{\mathrm{c} 3}=\left(s_{4}, 0.2\right)
\end{gathered}
$$

Following to:

$$
\left(\mathrm{m}_{\mathrm{i}}, \alpha_{\mathrm{i}}\right)=\Delta\left(\mathrm{g}\left(\sum_{\mathrm{j}}^{\mathrm{n}}\left(w_{j}\right) \cdot\left[1-\frac{\left|\Delta^{-1}\left(\mathrm{~S}_{\mathrm{ij}}, \alpha_{\mathrm{ij}}\right)-\Delta^{-1}\left(\mathrm{~T}_{\mathrm{j}}, \alpha_{\mathrm{j}}\right)\right|}{\mathrm{L}_{\mathrm{j}}-\mathrm{P}_{\mathrm{j}}}\right]\right)\right)
$$

Using the below operation for each criterion (example $\mathrm{C}_{3}$ ):

$$
\begin{aligned}
& \left(w_{\mathrm{c} 3}\right) \cdot\left[1-\frac{\left|\Delta^{-1}\left(S_{\mathrm{pc}, \mathrm{c} 3}, \alpha_{\mathrm{pc}, \mathrm{c} 3}\right)-\Delta^{-1}\left(T_{\mathrm{c} 3}, \alpha_{\mathrm{c} 3}\right)\right|}{L_{\mathrm{c} 3}-P_{\mathrm{c} 3}}\right] \Rightarrow \\
& 0.118 \times\left[1-\frac{\left|\left(s_{4}, 0.4\right)-\left(s_{5}, 0.4\right)\right|}{\left(s_{5}, 0.4\right)-\left(s_{4}, 0.2\right)_{c 3}}\right] \Rightarrow \\
& \Rightarrow 0.118 \times\left[1-\frac{1}{1.2}\right] \Rightarrow 0.118 \times\left[1-\frac{1}{1.2}\right]=0.02
\end{aligned}
$$

The sum of these operations will gathering in the final equation as following described:

$$
\begin{gathered}
\left(\mathrm{m}_{p c}, \alpha_{p c}\right)=\Delta\left(6\left(\sum_{\mathrm{j}}^{8}\left(w_{j}\right) \cdot\left[1-\frac{\left|\Delta^{-1}\left(\mathrm{~S}_{\mathrm{pc}, \mathrm{j}}, \alpha_{\mathrm{pc}, \mathrm{j}}\right)-\Delta^{-1}\left(\mathrm{~T}_{j}, \alpha_{\mathrm{j}}\right)\right|}{\mathrm{L}_{\mathrm{j}}-\mathrm{P}_{\mathrm{j}}}\right]\right)\right) \Rightarrow \\
\Rightarrow\left(\mathrm{m}_{p c}, \alpha_{p c}\right)=\Delta(6(0+0+0.02+0+0.023+0+0+0)) \Rightarrow \\
\Rightarrow\left(\mathrm{m}_{p c}, \alpha_{p c}\right)=\Delta(0.256) \Rightarrow\left(\mathrm{m}_{p c}, \alpha_{p c}\right)=\left(s_{0}, 0.256\right)
\end{gathered}
$$

The derivate rankings using the proposed method are the same that one produced by Jeya Girubha and Vinodh ${ }^{12}$ and by Liu et al. ${ }^{13}$. Thus, based on case study 1 results, the actual method is validated.

Table 2. Decision matrix- Case study 1 - Polymers selection.

\begin{tabular}{ccccccccc}
\hline \multirow{2}{*}{ Materials } & \multicolumn{7}{c}{ 2-tuple linguistic assessments of materials ${ }^{\mathrm{a}}$} \\
\cline { 2 - 8 } & $\mathrm{C} 1$ & $\mathrm{C} 2$ & $\mathrm{C} 3$ & $\mathrm{C} 4$ & $\mathrm{C} 5$ & $\mathrm{C} 6$ & $\mathrm{C} 7$ & $\mathrm{C} 8$ \\
\hline SMA & $\left(\mathrm{S}_{4}, 0,20\right)$ & $\left(\mathrm{S}_{5},-0,20\right)$ & $\left(\mathrm{S}_{4}, 0,20\right)$ & $\left(\mathrm{S}_{4}, 0,40\right)$ & $\left(\mathrm{S}_{4}, 0,20\right)$ & $\left(\mathrm{S}_{5},-0,40\right)$ & $\left(\mathrm{S}_{4}, 0,20\right)$ & $\left(\mathrm{S}_{4}, 0,20\right)$ \\
PC & $\left(\mathrm{S}_{4}, 0,00\right)$ & $\left(\mathrm{S}_{4},-0,20\right)$ & $\left(\mathrm{S}_{4}, 0,40\right)$ & $\left(\mathrm{S}_{4}, 0,20\right)$ & $\left(\mathrm{S}_{4}, 0,40\right)$ & $\left(\mathrm{S}_{4}, 0,00\right)$ & $\left(\mathrm{S}_{4},-0,40\right)$ & $\left(\mathrm{S}_{4},-0,20\right)$ \\
PP & $\left(\mathrm{S}_{6},-0,40\right)$ & $\left(\mathrm{S}_{6},-0,40\right)$ & $\left(\mathrm{S}_{5}, 0,40\right)$ & $\left(\mathrm{S}_{5}, 0,40\right)$ & $\left(\mathrm{S}_{5}, 0,40\right)$ & $\left(\mathrm{S}_{6},-0,40\right)$ & $\left(\mathrm{S}_{5}, 0,40\right)$ & $\left(\mathrm{S}_{5}, 0,20\right)$ \\
ABS & $\left(\mathrm{S}_{5}, 0,00\right)$ & $\left(\mathrm{S}_{6},-0,40\right)$ & $\left(\mathrm{S}_{5}, 0,40\right)$ & $\left(\mathrm{S}_{5}, 0,00\right)$ & $\left(\mathrm{S}_{5}, 0,40\right)$ & $\left(\mathrm{S}_{5}, 0,40\right)$ & $\left(\mathrm{S}_{6},-0,40\right)$ & $\left(\mathrm{S}_{5}, 0,00\right)$ \\
\hline
\end{tabular}

${ }^{\mathrm{a} A d a p t e d ~ f r o m ~ G i r u b h a ~ a n d ~ V i n o d h ~}{ }^{12}$.

Table 3. Results from obtained example by the proposed method (equations 9, 10 and 11) compared to earlier works.

\begin{tabular}{ccccc}
\hline Materials & Proposed Method & Proposed Method & Jeya Girubha and Vinodh & Liu et al. $^{13}$ \\
\hline SMA & $\left(\mathrm{S}_{1}, 0,34\right)$ & $3^{\mathrm{o}}$ & $3^{\mathrm{o}}$ & $3^{\mathrm{o}}$ \\
PC & $\left(\mathrm{S}_{0}, 0,26\right)$ & $4^{\mathrm{o}}$ & $4^{\mathrm{o}}$ & $4^{\mathrm{o}}$ \\
PP & $\left(\mathrm{S}_{6},-0,18\right)$ & $1^{\mathrm{o}}$ & $1^{\mathrm{o}}$ & $1^{\mathrm{o}}$ \\
ABS & $\left(\mathrm{S}_{5}, 0,27\right)$ & $2^{\mathrm{o}}$ & $2^{\mathrm{o}}$ & $2^{\mathrm{o}}$ \\
\hline
\end{tabular}


Case study 2 was adapted from Wang e Chang ${ }^{25}$. The adaptation consisted only in using linguistic data, which the authors develop the tool steel materials selection. In this case study, five types of AISI Tool Steel (W1, A2, $\mathrm{D} 2, \mathrm{~S} 1$, and $\mathrm{T} 1$ ) are alternatives to materials selection. The assessment criteria are as follow Nondeforming properties (C1); Safety in hardening (C2); Toughness (C3); Resistance to softening the effect of heat (C4); Wear resistance (C5); Machinability (C6); Material cost (C7).

The linguistic variables set used in the assessment of weight criteria, i.e., the relevance of each criteria was $\mathrm{S}=$ $\left\{\mathrm{S}_{0}=\operatorname{Very} \operatorname{Low}(\mathrm{VL}) ; \mathrm{S}_{1}=\operatorname{Low}(\mathrm{L}) ; \mathrm{S}_{2}=\operatorname{Medium}(\mathrm{M}) ; \mathrm{S}_{3}=\right.$ $\operatorname{High}(\mathrm{H}) ; \mathrm{S}_{4}=$ very High $\left.(\mathrm{VH})\right\}$.

The linguistic variables set used in the materials assessment, i.e., material performance was: $\mathrm{S}=\left\{\mathrm{S}_{0}=\right.$ Worst (W); $\mathrm{S}_{1}=$ Poor (P); $\mathrm{S}_{2}=$ Fair (F); $\mathrm{S}_{3}=\operatorname{Good}(\mathrm{G}) ; \mathrm{S}_{4}=$ Best (B)\}. The materials assessment was obtained from technical datasheets by qualitative assessments.
Table 4 shows the decision-makers assessments to weight criteria and the results of equation 6 and 7 applications, which comprehend the step 2 of the proposed method.

The assessments provided by three experts are presented in Table 5.

On development of case study 2, the Step 1 method was necessary to Criteria 7 - Cost qualitative values conversion. The relative cost data that were converted $(1,6 ; 2,0 ; 1,0$; $1,4 ; 3,0)^{\mathrm{T}}$ by equation 5 , the cost criteria resulted in follow linguistic variables set:( $,-0,50)$; ( $, 0,00)$; $(\mathrm{S}, 0,00)$; $(\mathrm{S},-0,14) ;(\mathrm{S}, 0,33)^{\mathrm{T}}$. The materials ${ }^{2}$ qualitative ${ }^{4}$ data are prẻsented at Táble 5 .

In step 4, one determines the intended goal to each criterion, in example 2 was used the alternative with better performance comparing to each criterion as well as the goal to be achieved. The goals set to seven criteria are as follow: $\mathrm{M}=\left\{\left(\mathrm{S}_{4}, 0,00\right) ;\left(\mathrm{S}_{4}, 0,00\right) ;\left(\mathrm{S}_{3}, 0,00\right) ;\left(\mathrm{S}_{4}, 0,00\right) ;\left(\mathrm{S}_{3}, 0,00\right) ;\right.$ $\left.\left(\mathrm{S}_{4}, 0,00\right) ;\left(\mathrm{S}_{4}, 0,00\right)\right\}$. Table 6 presents the ranking results obtained by the proposed method.

Table 4. Linguistic assessments of criteria weights ${ }^{\mathrm{a}}$, 2-tuple linguistic criteria weights and real numbers criteria weighs - Case study 2 - Tool steel selection.

\begin{tabular}{|c|c|c|c|c|c|c|c|}
\hline \multirow{2}{*}{ Weights } & \multicolumn{7}{|c|}{ Criteria } \\
\hline & $\mathrm{C} 1$ & $\mathrm{C} 2$ & $\mathrm{C} 3$ & $\mathrm{C} 4$ & $\mathrm{C} 5$ & C6 & $\mathrm{C} 7$ \\
\hline Decision-maker $1^{\mathrm{b}}$ & $\mathrm{H}$ & M & VH & $\mathrm{H}$ & M & $\mathrm{H}$ & VH \\
\hline Decision-maker 2 & $\mathrm{H}$ & $\mathrm{H}$ & VH & $\mathrm{H}$ & M & $\mathrm{H}$ & $\mathrm{VH}$ \\
\hline Decision-maker 3 & $\mathrm{VH}$ & M & $\mathrm{H}$ & M & M & VH & VH \\
\hline Equation (6) & $\left(\mathrm{S}_{3}, 0,33\right)$ & $\left(\mathrm{S}_{2}, 0,33\right)$ & $\left(\mathrm{S}_{4},-0,33\right)$ & $\left(S_{3},-0,33\right)$ & $\left(\mathrm{S}_{2}, 0,00\right)$ & $\left(\mathrm{S}_{3}, 0,33\right)$ & $\left(\mathrm{S}_{4}, 0,00\right)$ \\
\hline Equation (7) & 0,156 & 0,109 & 0,172 & 0,125 & 0,094 & 0,156 & 0,188 \\
\hline
\end{tabular}

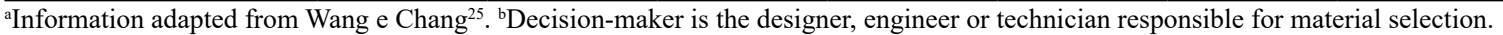

Table 5. Decision Matrix - Case study 2 - Tool Steel Selection

\begin{tabular}{cccccccc}
\hline \multirow{2}{*}{ Materials } & \multicolumn{7}{c}{ 2-tuple linguistic assessments of materials ${ }^{\mathrm{a}}$} \\
\cline { 2 - 8 } & $\mathrm{C} 1$ & $\mathrm{C} 2$ & $\mathrm{C} 3$ & $\mathrm{C} 4$ & $\mathrm{C} 5$ & $\mathrm{C} 6$ & $\mathrm{C} 7^{\mathrm{b}}$ \\
\hline $\mathrm{W} 1$ & $\left(\mathrm{~S}_{1}, 0,00\right)$ & $\left(\mathrm{S}_{2}, 0,00\right)$ & $\left(\mathrm{S}_{3}, 0,00\right)$ & $\left(\mathrm{S}_{1}, 0,00\right)$ & $\left(\mathrm{S}_{2}, 0,00\right)$ & $\left(\mathrm{S}_{4}, 0,00\right)$ & $\left(\mathrm{S}_{3},-0,50\right)$ \\
A2 & $\left(\mathrm{S}_{4}, 0,00\right)$ & $\left(\mathrm{S}_{4}, 0,00\right)$ & $\left(\mathrm{S}_{2}, 0,00\right)$ & $\left(\mathrm{S}_{2}, 0,00\right)$ & $\left(\mathrm{S}_{3}, 0,00\right)$ & $\left(\mathrm{S}_{2}, 0,00\right)$ & $\left(\mathrm{S}_{2}, 0,00\right)$ \\
D2 & $\left(\mathrm{S}_{4}, 0,00\right)$ & $\left(\mathrm{S}_{4}, 0,00\right)$ & $\left(\mathrm{S}_{2}, 0,00\right)$ & $\left(\mathrm{S}_{2}, 0,00\right)$ & $\left(\mathrm{S}_{3}, 0,00\right)$ & $\left(\mathrm{S}_{1}, 0,00\right)$ & $\left(\mathrm{S}_{4}, 0,00\right)$ \\
S1 & $\left(\mathrm{S}_{2}, 0,00\right)$ & $\left(\mathrm{S}_{3}, 0,00\right)$ & $\left(\mathrm{S}_{3}, 0,00\right)$ & $\left(\mathrm{S}_{2}, 0,00\right)$ & $\left(\mathrm{S}_{2}, 0,00\right)$ & $\left(\mathrm{S}_{2}, 0,00\right)$ & $\left(\mathrm{S}_{3},-0,14\right)$ \\
T1 & $\left(\mathrm{S}_{3}, 0,00\right)$ & $\left(\mathrm{S}_{3}, 0,00\right)$ & $\left(\mathrm{S}_{2}, 0,00\right)$ & $\left(\mathrm{S}_{4}, 0,00\right)$ & $\left(\mathrm{S}_{3}, 0,00\right)$ & $\left(\mathrm{S}_{2}, 0,00\right)$ & $\left(\mathrm{S}_{1}, 0,33\right)$ \\
\hline
\end{tabular}

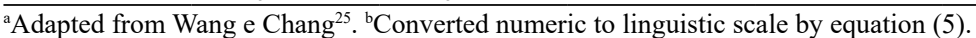

Table 6. Case study 2 results obtained by the proposed method, Equations 9,10 and 11 application compared to earlier work.

\begin{tabular}{cccc}
\hline Material & Proposed Method & Proposed Method & Wang and Chang $^{25}$ \\
\hline W1 & $\left(\mathrm{S}_{2}, 0,36\right)$ & $5^{\mathrm{o}}$ & $5^{\mathrm{o}}$ \\
A2 & $\left(\mathrm{S}_{2}, 0,00\right)$ & $2^{\mathrm{o}}$ & $1^{\mathrm{o}}$ \\
D2 & $\left(\mathrm{S}_{2}, 0,35\right)$ & $1^{\mathrm{o}}$ & $2^{\mathrm{o}}$ \\
S1 & $\left(\mathrm{S}_{2},-0,08\right)$ & $3^{\mathrm{o}}$ & $4^{\mathrm{o}}$ \\
T1 & $\left(\mathrm{S}_{2},-0,28\right)$ & $4^{\mathrm{o}}$ & $3^{\mathrm{o}}$ \\
\hline
\end{tabular}


According to Table 6, the derivate ranking using the proposed method differs from the obtained by Wang and Chang ${ }^{25}$. According to Rao and Patel ${ }^{15}$ do not matter if different methods provide different rankings, once the first place material is consistent, which occurs in case study 2 since the D2 steel is tool steel widely used in the fabrication of cold-work dies.

\section{Conclusions}

The proposed material selection method allows process quantitative and qualitative information as linguistics variables, as well as in different linguistic variable scales, enables the identification of the intended goal to materials selection in the ranking step.

The results obtained from proposed method application in materials selection issues showed agreement to results obtained by earlier researchers with linguistics data processed by fuzzy and 2-tuple, case study 1 and solution consistency, despite material selected difference, case study 2 .

The proposed method is computationally simple and allows processing of materials selection issues with uncertainty in information modeled by linguistic variables.

\section{References}

1. Ashby MF. Materials selection in mechanical design. $M R S$ Bulletin. 2005;30(12):995.

2. Ashby MF, Bréchet $\mathrm{Y}$, Cebon D. Selection strategies for materials and processes. Advanced Enginnering Materials. 2002;4(6):51-67.

3. Lovatt AM, Shercliff HR. Manufacturing process selection in engineering design. Part 1: The role of process selection. Materials and Design. 1998;19(5-6):205-215.

4. Jahan A, Ismail MY, Sapuan SM, Mustapha F. Material screening and choosing methods - A review. Materials and Design. 2010;31:696-705.

5. Kumar R, Singal SK. Penstock material selection in small hydropower plants using MADM methods. Renewable and Sustainable Energy Reviews. 2015;52:240-255.

6. Chauhan A, Vaish R. Hard coating material selection using multicriteria decision making. Materials and Design. 2013;44:240245.

7. Mousavi-Nasab SH, Sotoudeh-Anvari A. A comprehensive MCDM-based approach using TOPSIS, COPRAS and DEA as an auxiliary tool for material selection problems. Materials and Design. 2017;121:237-253.

8. Antunes RA, Salvador CAF, Oliveira MCL. Materials selection of optimized titanium alloys for aircraft applications. Materials and Design. 2018;21(2):e20170979. DOI: https:// doi.org/10.1590/1980-5373-mr-2017-0979

9. Dehghan-Manshadi B, Mahmudi H, Abedian A, Mahmudi R. A novel method for materials selection in mechanical design: Combination of non-linear normalization and a modified digital logic method. Materials and Design. 2007;28(1):8-15.
10. Milani AS, Shanian A, Madoliat R, Nemes JA The effect of normalization norms in multiple attribute decision making models: a case study in gear material selection. Structural and Multidisciplinary Optimization. 2005;29(4):312-318.

11. Sarfaraz R, Manshadi BD, Abedian A, Mahmudi R. A simplified fuzzy logic approach for materials selection in mechanical engineering design. Materials and Design. 2009;30(3):687-697.

12. Girubha RJ, Vinodh S. Application of fuzzy VIKOR and environmental impact analysis for material selection of an automotive component. Materials and Design. 2012;37:478-486.

13. Liu HC, Liu L, Wu J. Material selection using an interval 2-tuple linguistic VIKOR method considering subjective and objective weights. Materials and Design. 2013;52:158-167.

14. Fayazbakhsh K, Abedian A, Manshadi BD, Khabbaz RS. Introducing a novel method for materials selection in mechanical design using Z-transformation in statistics for normalization of material properties. Materials and Design. 2009;30(10):4396-4404.

15. Rao RV, Patel BK. A subjective and objective integrated multiple attribute decision making method for material selection. Materials and Design. 2010;31(10):4738-4747.

16. Xu Z. Linguistic decision making: theory and methods. Nanjing, Jiangsu, China: Springer-Verlag Berlin Heidelberg, Institute of Sciences, PLA University of Science and Technology Nanjing; 2012. DOI: https://doi.org/10.1007/978-3-642-29440-2

17. Herrera F, Martínez L. A 2-tuple fuzzy linguistic representation model for computing with words. IEEE Transactions on Fuzzy Systems. 2000;8:746-752.

18. Xu Z. Linguistic aggregation operators. In: Xu Z, editors. Linguistic decision making: theory and methods. Berlin, Heidelberg: Springer-Verlag Berlin Heidelberg; 2012. p. 15-85.

19. Hwang CL, Yoon K. Multiple attribute decision making: methods and applications a state-of-the-art suvery. Berlin, Heidelberg: Springer-Verlag Berlin Heidelberg; 1981. DOI: https:/doi.org/10.1007/978-3-642-48318-9

20. Zanakis SH, Solomon A, Wishart N, Dublish S. Multiattribute decision making: a simulation comparison of select methods. European Journal of Operational Research. 1998;107(3):507-529.

21. Herrera F, Herrera-Viedma E, Martinez L. A fuzzy linguistic methodology to deal with unbalanced linguistic term sets. IEEE Transactions on Fuzzy Systems. 2008;16(2):354-370.

22. Martínez L, Herrera F. An overview on the 2-tuple linguistic model for computing with words in decision making: extensions, applications and challenges. Information Sciences. 2012;207:1-18.

23. Martínez L, Liu J, Yang JB, Herrera F. A multigranular hierarchical linguistic model for design evaluation based on safety and cost analysis. International Journal of Intelligent Systems. 2005;20:1161-1194.

24. Jahan A, Bahraminasab M, Edwards KL. A target-based normalization technique for materials selection. Materials and Design. 2012;35:647-654.

25. Wang MJJ, Chang TC. Tool steel materials selection under fuzzy environment. Fuzzy Sets and Systems. 1995;72(3):263-270. 